\title{
Management of obstructive sleep disordered breathing in 2 to 18 years old children from ERS perspective
}

\author{
Sorina Chindris ${ }^{1,2}$, Doina-Anca Plesca ${ }^{1,2}$ \\ ${ }^{1}$ Faculty of Medicine, "Carol Davila" University of Medicine and Pharmacy, Bucharest, Romania \\ 2Paediatric Department, "Victor Gomoiu“ Children's Hospital, Bucharest, Romania
}

\begin{abstract}
Sleep related breathing disorders in children and adolescents represent a new pathology both in terms of diagnostic mechanisms and therapeutic measures that are in a continuous multidisciplinary approach.

Experts from the European Respiratory Society have tried to establish a guideline in the evaluation and management of children with obstructive sleep disorders (OSA). In this article we will address the pediatric population from 2 to 18 years old.

OSA are defined by the presence of specific signs and symptoms of partial or complete obstruction of the upper airway, both of which occur during sleep.

Polysomnography is considered the gold standard of SDB evaluation. Thus, an apnea:hypopnea index $>5$ events / hour or an apnea:hypopnea index $>1$ event / hour but in the presence of risk factors or associated comorbidities, requires specific treatments.

Adenotonsillectomy remains the first line of treatment for OSA in children with adenotonsillar hypertrophy. Interventional measures are extremely varied. We can use oral therapeutic devices, noninvasive ventilation, drug therapy or surgery. Each interventional measure will be individualized in order to improve the quality of life of every patient. Our aim is to obtain clinical improvement or cure and to treat any complications that may appear over time. All these complex aspects bring together doctors from different specialties to provide patients with the most effective medical care services.
\end{abstract}

Keywords: sleep disordered breathing, sleep apnea, polysomnography, noninvasive ventilation, child

\section{INTRODUCTION. TERMINOLOGY. EPIDEMIOLOGY}

Sleep together with waking activities are important elements in the functioning of the human body.

During sleep, essential processes take place with a role in the harmonious development of a child, with psychomotor and neurobehavioural acquisitions. Sleep has an important role in the secretion of growth hormones, regulation of body temperature, memory and learning, creativity and modeling of emotional functions, improving immune activity, brain development etc. (1).

Sleep is defined as a natural, dynamic, physiological activity that occurs periodically, in which the body rests and the state of consciousness is suspended (2).
Obstructive sleep respiratory disorders in children (OSA) occur secondary to upper airway dysfunction during sleep.

Sleep-related breathing disorders in children are a broad clinical spectrum of pathophysiological manifestations characterised by partial or complete obstruction of the upper airways, events that occur during sleep.

The literature describes several clinical forms of sleep-related breathing disorders that occur in sleep. Therefore the following can be found: primary snoring, upper airway resistance syndrome, obstructive sleep apnea with various forms of severity.

Primary snoring is defined by the presence of frequent snoring, more than 3 times a week, which does not wake up the child. Primary snoring does not as- 
sociate an obvious pathology of the upper airways and has no major respiratory consequences (apnea, hypoventilation, hypercapnia or other sleep disorders) (3).

Upper airway resistance syndrome is a respiratory disorder that occurs during sleep and combines increased inspiratory effort, snoring and possible clinical features (sleep fragmentation, daytime sleepiness, fatigue, restlessness). It is associated with arousals, a decrease in oxygen saturation up to $3 \%$ but no obstructive events are recorded (3).

Obstructive sleep apnea is a pathological condition associated with repeated episodes of partial or complete upper airway obstruction that occur during sleep and it has a duration of at least $10 \mathrm{sec} / 2$ respiratory cycles. This clinical form associates inspiratory effort during sleep and causes ventilation disorders (3).

The most common symptom of sleep-related breathing disorders in children is snoring. It occurs with a prevalence of almost $7.45 \%$ amongs children. (4) The prevalence of SASO amongs pediatric population ranges between 0.1 and $13 \%$. It occurs in all age groups, from newborn to teenager, with higher incidence seen in preschool age (4).

\section{PATHOPHYSIOLOGY}

There are at least 4 pathophysiology mechanisms leading to manifestations of sleep-related breathing disorders in children $(1,5)$ :

- Adenotonsillar hypertrophy - it is considered the most common cause of OSA in children. The increasing volume of the tonsills / adenoid hypertrophy may produce the collapse of the upper airway during sleep, with varying degrees of air flow restriction.

- Craniofacial anatomy - craniofacial dysmorphism or craniofacial malformations are another common cause of OSA in children. Changes in ventilation are directly proportional to the child's age and changes in the bone structure.

- Respiratory muscle damage that occurs in the group of neuromuscular diseases, genetic and metabolic diseases. The absence or the decrease in the respiratory muscular support is secondary to the progressive muscular atrophy and to the decrease in the dinamics of the thoracic cave. All these changes cause different degrees of upper airways obstruction and followed by hypoxia, hypoventilation, hypercapnia.

- Obesity is an important cause of OSA in the pediatric population through the clinical implications and metabolic consequences it has upon the whole body.

\section{CLINICAL MANIFESTATIONS}

The symptoms of sleep-related breathing disorders are complex. They can be clinically expressed through a wide range of signs and symptoms that occur both during sleep and in wakefulness: restless or fragmented sleep, snoring, bedwetting, nightmares, morning headache, daytime drowsiness, aggression, difficulty in concentrating or learning, speech disorders, symptoms in the spectrum of attention deficit, growth disorders, cardiovascular signs and symptoms (hypertension, arrhythmias etc.) (6).

\section{DIAGNOSIS AND MANAGEMENT}

At this moment there are several techniques for screening and diagnosing obstructive sleep respiratory disorders: nocturnal pulsoximetry, cardiorespiratory polygraphy, polysomnography, video recordings, actigraphy etc.

Polysomnography (PSG) is considered the "goldstandard" method for OSA diagnosis. Polysomnography is a complex and laborious investigation that requires time, physical and financial resources. The investigation provides important informations on sleep architecture, neurological and cardiorespiratory events associated with sleep.

The polysomnographic pathway includes monitoring of neurological and cardiorespiratory parameters during sleep. The evaluation of neurological parameters and sleep structure is done by using electroencephalogram, electrooculogram, electromyogram, leg movements etc. Cardiorespiratory parameters sum up information from the oronasal flow sensors, the thoracic and abdominal belt movements, pulsoximetry, cardiac activity, nocturnal monitoring of carbon dioxide values etc.

Experts from the Scientific Committee of the $\mathrm{Eu}-$ ropean Respiratory Society agreed to a protocol for the diagnosis and management of obstructive sleep respiratory disorders in children. It contains several steps that validate the evaluation path of a pediatric patient aged between 2 and 18 years with sleep-related breathing disorders. The algorithm takes into account the entire history and clinical manifestations as well as associated comorbidities, in order to ensure the appropiate management strategies for each child (7).

Table 1 presents the main parameters to take into consideration when obstructive sleep respiratory disorders are suspected in children. These parameters are correlated with the risk factors and the associated comorbidities as well as with the predictable factors for persistence of OSA. The main purpose of this evalua- 


(1)

FIGURE 1. Polysomnography in children - the epoch shows N2 stage of sleep, without obstructive respiratory events, oxygen saturation within normal limits (personal collection)

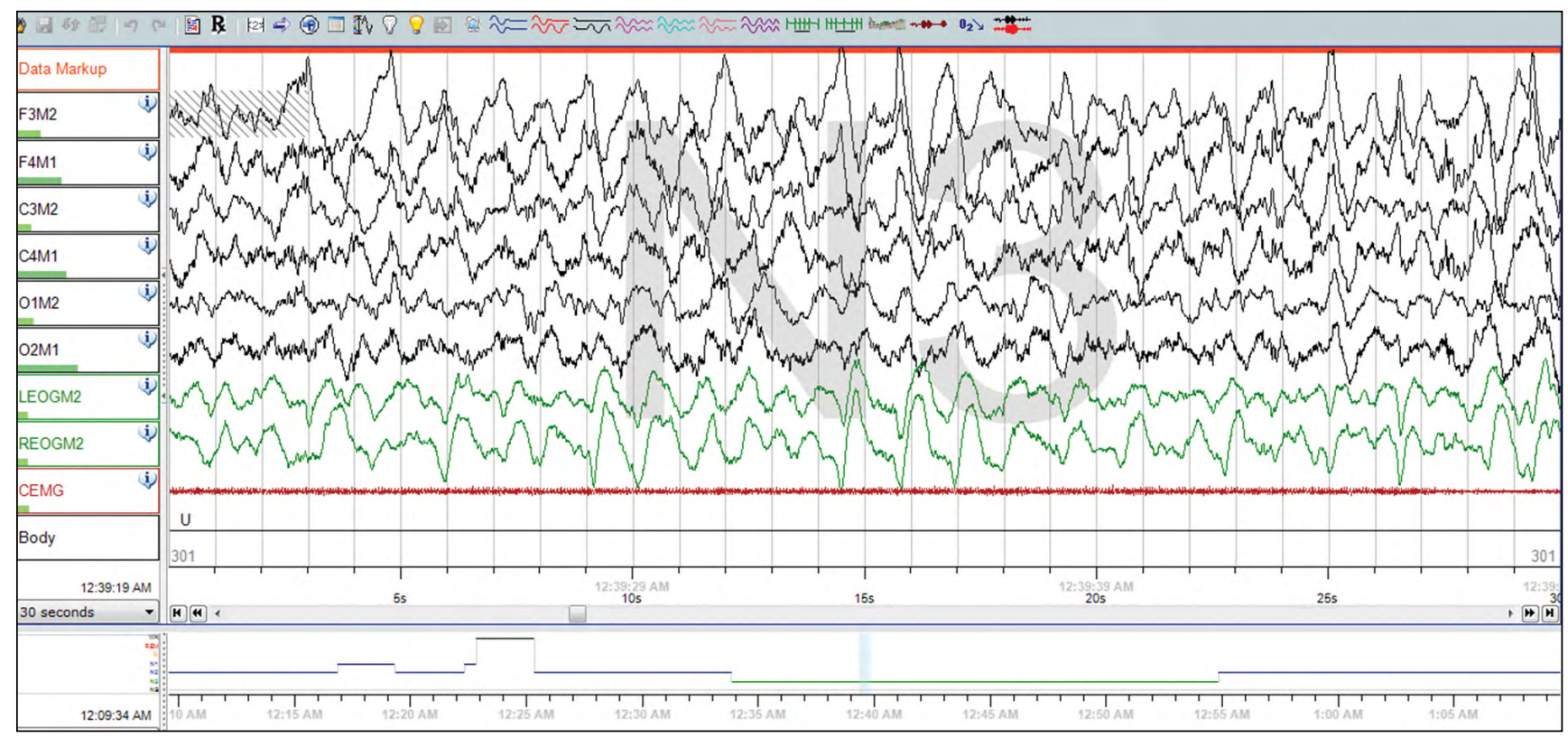

FIGURE 2. Polysomnography in children: Stage N3 sleep - personal collection 


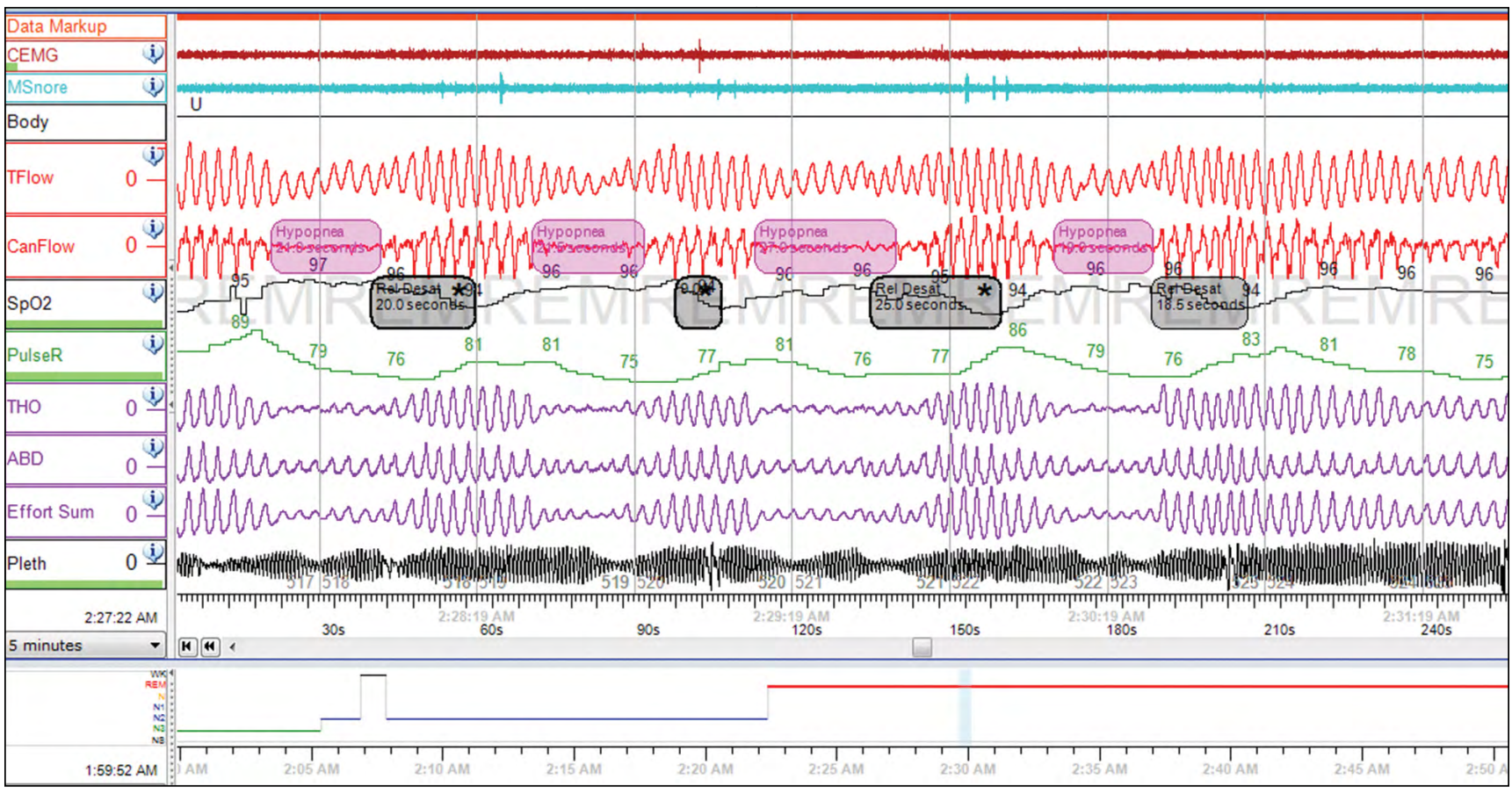

FIGURE 3. Polysomnography in children: Obstructive hypopnea in the REM stage - personal collection

TABLE 1. Evaluation of parameters for the development of OSA in children with obstructive sleep respiratory disorders adapted by Khaditis $A$ et al. (7)

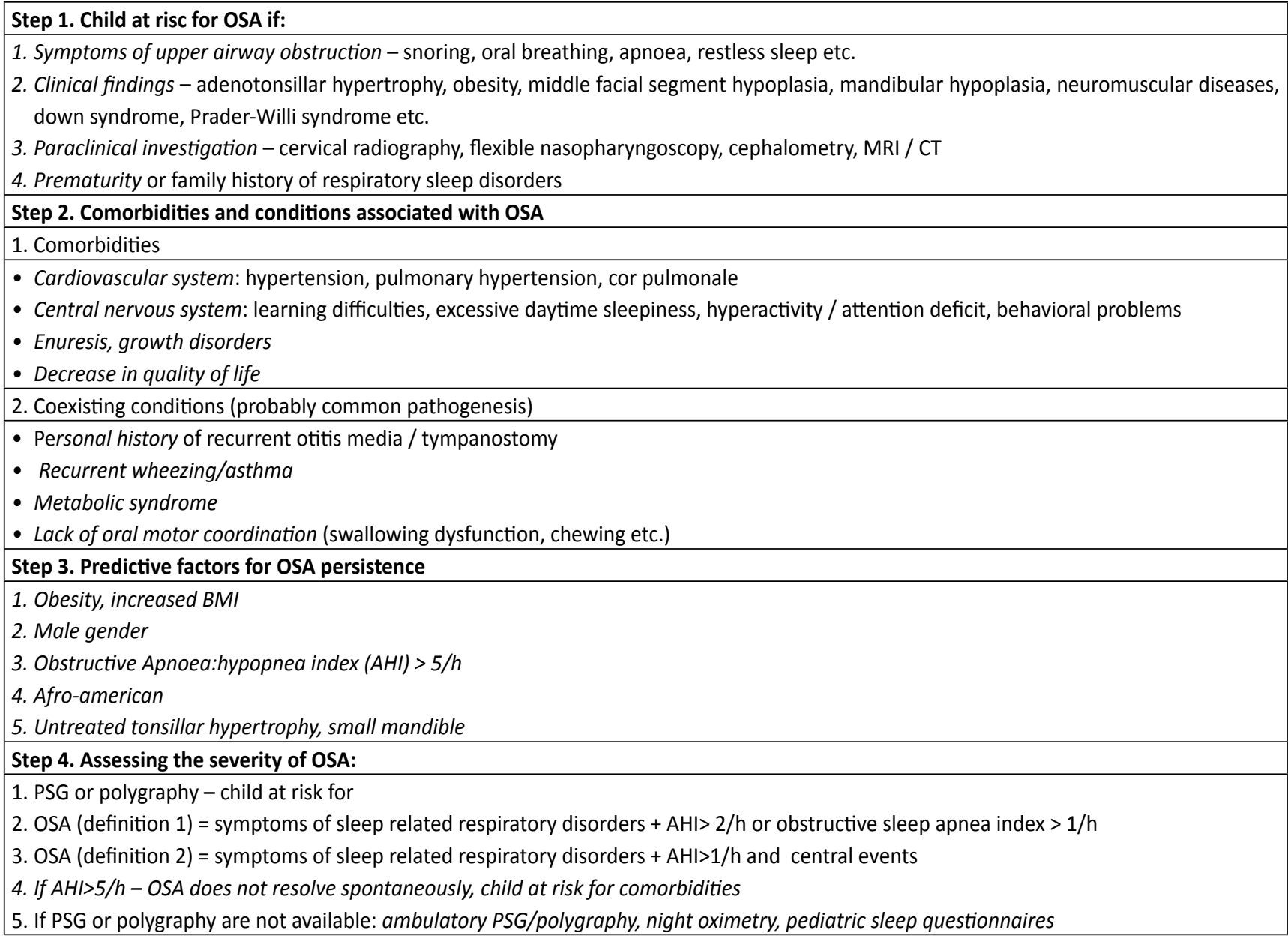


tion is to establish the severity of OSA using objective diagnostic investigation mechanisms.

The literature provides a variety of informations about the impact that comorbidities associated with OSA have on various systems and organs. Comorbidities can become a trigger, can maintain or worsen the pathology of OSA amongs the paediatric population.

Studies in the literature show that the prevalence of OSA amongs the obese paediatric population varies between $13 \%$ and $59 \%$ (8). The percentage increases with the degree of obesity (9). Metabolic syndrome and OSA are 2 entities commonly associated with obesity. Lossing weight is a life strategy that improves both pathologies (7).

Neuromuscular diseases are associated with an evolving form of sleep-related respiratory disorders. The severity of OSA is dependent on the age of onset, the type of neuromuscular disease, the degree of respiratory muscle damage and the patient's compliance with treatment.

The study conducted by Mellies et al. (10) shows that children diagnosed with neuromuscular diseases that associate a decrease in inspiratory vital capacity $<$ $60 \%$, these values become a negative prognostic factor associated with the presence of OSA and a decrease $<40 \%$ may predict the association of OSA with nocturnal hypoventilation.

Obstructive sleep respiratory disorders may occur even in the absence of diurnal or nocturnal symptoms observed by the patient or entourage (8).

The pediatric sleep questionnaire is validated as a tool for assessing sleep-related respiratory disorders in children (9). The questionnaire applies to the patient and points out important signs and symptoms for the development of this pathology: the presence of snoring, apnoea, daytime sleepiness, waking up difficulties, headache, lack of concentration, lack of attention, difficulties in memorizing and performing various tasks, restlessness, involuntary urine emissions, changes in growth rate etc. Depending on the score obtained, the questionnaire rises the suspicion of a possible sleep-related respiratory disorder and the investigations are supplemented according to the patient's pathology (9).

Cardiorespiratory polysomnography is considered the standard method for diagnosing OSA at any age.

The American Academy of Pediatrics and the American Academy of Sleep Medicine recommend attended polysomnography foe evaluating OSA, performed in the sleep laboratory, under medical supervision (7). The other accepted OSA investigation is cardiorespiratory polygraphy (7).

Polysomnography performed during daytime sleep, outpatient polysomnography, nighttime oximetry or video recordings may be proposed as alternative methods for assessing sleep disorders if standard polysomnography is not accessible (7).

Polysomnography or polygraphy is recommended for children with symptomatic OSA and prior to adenotonsillectomy, especially in the presence of comorbidities: obesity, neuromuscular or other genetic and metabolic diseases, craniofacial dysmorphism (7).

Polysomnography or polygraphy is recommended postadenotonsillectomy in children who maintain persistent OSA or have a moderate-severe form of OSA prior to surgery and in children who associate the comorbidities mentioned above (7).

Polysomnography or polygraphy have indication before and after application of orthodontic devices, after maxillar expansion surgery as well as for establishing the parameters of noninvasive ventilation with continuous positive pressure (7).

The rules for interpreting polysomnographic parameters of recorded sleep are well defined by the American Academy of Sleep Medicine (AASM) through up to date textbooks.

Apnoea:hypopnoea index is the number of respiratory events recorded during sleep (mixed, obstructive or central apnoea / hypopnoea) related to the total time of sleep hours and is expressed in number of events per hour. This index is one of the most widely used parameter of polysomnography in the interpretation of OSA severity (12).

Considering the history, the clinical examination and the investigations results performed individually for each child, we can establish the severity of OSA as well as the medium and long term consequences of this pathology upon the body. Polysomnographic results and clinical condition of the patient decide how to best approach both respiratory and related pathology.

Table 2 presents the main changes to take into consideration when it comes to the necessary investigations for the possible therapeutic approaches, the follow-up strategies both in case of a favorable evolution or in case of OSA persistence.

Starting treatment in a child with OSA depends on IAH values and on the complex pathology of the child. Thus, even if the PSG result shows an AHI between 1-5 / hour, treatment can be initiated if the patient has an increased risk of morbidity due to cardiovascular/other risk factors or if the quality of life is altered (7).

The treatment of OSA in a child includes measures to improve sleep quality associated with drug therapy, interventional therapy or noninvasive ventilation with continuous positive pressure.

Educational Strategies for Good Sleep Hygiene (AASM) (13) are as follows: 
TABLE 2. Management of SASO in children with obstructive sleep respiratory disorders - adapted by Khaditis AG et al. (7)

\begin{tabular}{|l|}
\hline Step 5 . Treatment approach of OSA \\
\hline 1. AHI $>5 / h,+/$ - comorbidities \\
2. AHI $<5 / h+$ cardiovascular comorbidities / CNS, enuresis, growth disorders, decreased quality of life, presence of predictive risk factors \\
3. Annual reassessment of primary snoring \\
4. Priority: presence of craniofacial abnormalities, neuromuscular diseases, achondroplasia, Chiari malformation, Down syndrome, MPZ, Prad- \\
er-Willi syndrome \\
\hline Step 6. Treatment options \\
\hline 1. Sleep hygiene \\
2. Weight loss - overweight/obese \\
3. Topical nasal or oral corticosteroid medication \\
4. Adenotonsillectomy \\
5. Orthodontic devices, maxillary expansion \\
6. Noninvasive ventilation \\
7. Craniofacial surgery \\
8. Traheostomy \\
\hline Step 7. Recognition and management of persistent OSA \\
1. Periodic monitoring at 6 weeks-12 months: symptoms, PSG, quality of life, comorbidities \\
2. PSG of choice for residual OSA detection. If PSG is not available: polygraphy, oximetry, capnography \\
3. PSG at 6 weeks after adenotonsillectomy if OSA persists or if the child is at risk \\
4. PSG at 12 weeks after oral or intranasal corticosteroid treatment \\
5. PSG at 12 months after maxillary expansion (faster if symptoms persist) and at 6 months after appliance of oral devices \\
6. PSG in order to initiate or maintain nocturnal ventilation with positive continuous pressure \\
7. Reassessment of the upper airway by nasopharyngoscopy, endoscopy, MRI \\
\hline
\end{tabular}

- Sleep ritual (same hours for sleeping and waking);

- Bedroom away from noise, cold and dark room;

- Avoid excess fluids before bed;

- voiding stimulating/exciting events before bedtime (activities, caffeine, stimulant medication etc.)

- Meal time hours

- Sleep hours - sufficient need for sleep

- Sleeping position (lying on the side, reclining position of the bed)

- Vocal exercises to stimulate the neck muscles

Medication administered to relieve the symptoms of OSA may include: nasal saline, nasal decongestant, antihistamines (in case of allergic rhinitis), corticosteroid therapy (intranasal/oral), oral antileukotriene therapy etc. (6).

In a study published in 2012, Goldbart AD et al. conclude that the administration of intranasal or oral corticosteroids to children with moderate-severe OSA for a period of 6-12 weeks reduces the severity of OSA, IAH and adenoids size but with poorer effects on the obese pediatric population (14).

Adenotonsillectomy is the surgical indication for children with OSA who associate adenotonsillar hypertrophy. Surgery improves cardiovascular outcomes, improves neurobehavioral symptoms and the quality of life (13).

The most common surgical approaches are the following:
- adenotonsillectomy

- tonsillotomy

- tracheostomy

- plastic: uvulopalatopharyngoplasty, uvulopalatoplasty

- radiofrequency ablation at the level of the soft palate

- soft palate implant etc.

Noninvasive ventilation with continuous positive pressure is addressed to the children with residual OSA postadenotonsillectomy, OSA associated with obesity, craniofacial abnormalities or neuromuscular diseases and in case of failure of other therapeutic options.

Noninvasive ventilation therapy improves gas exchange, symptoms (drowsiness, sleep-related disorders, behavioral disorders etc.) and thus improves the patient's quality of life. Adherence to noninvasive ventilation therapy varies and depends on symptom relief, family availability, education, perception and understanding of the treatment's benefits (2).

In children with mild OSA or with low compliance for noninvasive ventilation with positive continuous pressure, anti-snoring devices can be used to relieve symptoms and to improve the quality of life in this children. Anti-snoring devices that can be used are : nasal devices (nasal strips, nasal dilators), oral devices (chin strips, mouth devices), mandibular repositioning devices (7).

The lack of a personalized treatment (depending on the child's history and the results of investiga- 
tions), leads in time to the installation of complications derived from the progression of the basic disease or from the sleep-related respiratory disorders.

A good therapeutic response involves permanent doctor-patient communication, correct treatment measures, understanding the treatment and a good compliance, well-established periodic follow-ups and early recognition of possible complications of the disease/prescribed treatment.

\section{CONCLUSIONS}

Obstructive sleep respiratory disorders in children are a challenge for experts in this field. Multidiscipli- nary teams come together to understand the mechanisms underlying this pathology in order to find new treatment resources and new ways to prevent it.

The doctor's role is to acquire as much knowledge in the field in order to provide the child with an accurate clinical and paraclinical assessment and to decide an individualized therapeutic strategy. The doctor must focuse on the individual characteristics of every child in correlation with genetics, pathophysiology and environment factors that are involved.

Therefore we can create an unic individual, complex, varied, defined person, a challenge for current and future medical generations that try to understand, to find answers and solutions for a better preservation of this whole unit.

\section{REFERENCES}

1. Simonds AK, Backer W et al. ERS Handbook. Respiratory Sleep Medicine. Page Bros. UK. 2012; 205-210.

2. Spriggs WH. Essentials of polysomnography. A training guide and reference for sleep technicians. Jones \& Bartlett Learning, 2014.

3. Liukkonen K. Snoring and sleep apnea in children. University of Helsinki, Faculty of Medicine, Institute of Clinical Medicine, 2011.

4. Lumeng JC, Chervin RD. Epidemiology of Pediatric Obstructive Sleep apnea. Proc Am Thorac Soc. 2008;5(2):242-252.

5. Plesca DA, Chindris S, Avramuta A. Obesity and obstructive sleep apnea in children. The Publishing House of the Romanian Academy. 2015;1:3-5.

6. Cataletto ME, Serebrisky D et al. Childhood Sleep Apnea - Available at https://emedicine.medscape.com/article/1004104-overview.

7. Kaditis AG, Alvarez ML et al. Obstructive sleep disordered breathing in 2 to 18 -year-old children: Diagnosis and management. Eur Respir J. 2016;47:69-94.

8. New Zealand Guidelines for the Assessment of Sleep-Disordered Breathing in Childhood. Paediatric Society of New Zealand, 2015.

9. Oros M. Tulburarile de somn la sugar, copil mic si adolescent. Medichub, 2017.

10. Mellies U, Ragette R, Schwake C et al. Daytime predictors of sleep disordered breathing in children and adolescents with neuromuscular disorders. Neuromuscul Disord. 2003;13:123-128.

11. Suresh $S$, Wales $P$, Dakin $C$ et al. Sleep-related breathing disorder in Duchenne muscular dystrophy: Disease spectrum in the paediatric population. J Paediatr Child Health. 2005;41:500-503.

12. Spriggs WH. Essentials of Polysomnography. Jones \& Bartlett Learning. 2014;100-328.

13. Iber $C$ et al. The AASM manual for the scoring of sleep and associated events : rules, terminology and technical specifications. American Academy of Sleep Medicine. 2007;1-80.

14. Goldbart AD, Greenberg-Dotan S, Tal A. Montelukast for children with obstructive sleep apnea: A double-blind, placebo-controlled study. Pediatrics 2012;130:575-80. 